\title{
Acanthosis nigricans in a Chinese girl with FGFR3 K650 T mutation: a case report and literature review
}

\author{
Junling Fu', Yiting Zhao ${ }^{2}$, Tong Wang ${ }^{1}$, Qian Zhang ${ }^{1}$ and Xinhua Xiao ${ }^{1 *}$ (D)
}

\begin{abstract}
Background: Acanthosis nigricans (AN) is a clinical manifestation featured by velvety brown plaques in skin folds that occurs in some hereditary and syndromic disorders. Fibroblast growth factor receptor 3 (FGFR3) mutations have been identified as one of the genetic causes of inherited AN.

Case presentation: A 17-year-old Chinese female had presented generalized acanthosis nigricans since she was 4 years old. She yielded no family history of short stature or AN. Apart from a short stature, no skeletal defects, neurological defects or other abnormalities were found. To identify the aetiology of the clinically diagnosed AN, we screened the proband for genetic mutations using whole exome sequencing. A heterozygous mutation (c.1949A > C, p.Lys650Thr) in FGFR3 was found in the proband. To date, 26 cases of AN harbouring this specific gene mutation have been reported in the literature, and only one child carried a de novo mutation instead of inheriting the specific mutation from their parents. The present case is the first-reported Chinese patient with isolated AN with a de novo K650 T mutation in FGFR3.
\end{abstract}

Conclusions: We reported a new case of AN caused by a heterozygous mutation (c.1949A > C, p.K650 T) in FGFR3, and review the past reports of AN with the same gene mutation. Sequencing of the FGFR3 gene is a feasible approach to identify the aetiology of AN, especially for early onset extensive AN.

Keywords: Acanthosis nigricans, FGFR3, Mutation

\section{Background}

Acanthosis nigricans (AN) is characterized by velvety and pigmented hyperkeratosis of the skin, primarily in the skin folds of the neck, armpits, and groin. AN can occur as a symptomatic state of several genetic diseases, many of which are caused by insulin resistance syndromes or functional aberration of FGFR [1]. Mutations in fibroblast growth factor receptor 3 (FGFR3) are known to cause several kinds of skeletal dysplasia accompanying AN [2-5].

FGFR3 encodes a member of the fibroblast growth factor receptor (FGFR) family which is comprised of four related receptors (FGFR1-4) [2]. Different mutations in FGFR3 have been identified in patients with hypochondroplasia $(\mathrm{HCH})[2-5]$. Additionally, AN has occasionally been

\footnotetext{
* Correspondence: xiaoxh2014@vip.163.com

${ }^{1}$ Department of Endocrinology, Chinese Academy of Medical Sciences and Peking Union Medical College, Peking Union Medical College Hospital, Beijing, China

Full list of author information is available at the end of the article
}

reported in patients with mutations in FGFR3 [1-6]. In 2007, the first familial case of AN was reported with heterozygous mutation at codon 650 (p. Lys650Thr) in FGFR3 [6]. Presently, there have been 6 reports, totaling 26 cases, that have shown this specific gene mutation in $\mathrm{AN}$, and only one child carried a de novo mutation instead of inheriting the specific mutation from their parents [1-6]. In this study, we describe the first Chinese clinically diagnosed AN case with a de novo FGFR3 mutation (p. Lys650Thr), and review the previous reports of AN associated with activating mutations of FGFR3.

\section{Case presentation}

A 17-year-old girl was referred to our endocrinology clinic for hyperkeratotic and pigmented lesions on her neck and whole trunk, which initially appeared when she was 4 years old. Her height was within the normal range during childhood $(<4$ years $)$ but gradually began 
to be under the normal growth curve, ultimately resulting in grown-up short stature.

The patient was the first child of an unrelated Chinese couple. Her mother underwent vaginal delivery after a full-term pregnancy. The birth weight of the girl was 4 $\mathrm{kg}$ and the birth length was $50 \mathrm{~cm}$. She exhibited no neurological defects or skeletal abnormalities, no diabetes mellitus or its related symptoms, and no family history of cancer. The patient's parents, younger sister and brother had no significant medical history (Fig. 1).

On physical examination, the patient exhibited extensive, velvety, thick, hyperpigmented plaques involving the neck, back, and axillae (Fig. 2). The patient was a non-dysmorphic girl with the height of $146 \mathrm{~cm}(<-2 \mathrm{SD})$.

Laboratory tests revealed no abnormal biochemical findings (Table 1). The thyroid hormone, cortisol and androgen levels were within the normal range (the testosterone level demonstrated in Table 1 was under the reference range, we tested testosterone one more time, and the other value was normal: $31.8 \mathrm{ng} / \mathrm{dl}$ ). Fasting blood glucose and fasting insulin level were $88.2 \mathrm{mg} / \mathrm{dL}$ and $13.78 \mu \mathrm{U} / \mathrm{ml}$, respectively. The homeostasis assessment index for insulin resistance (HOMA-IR) as the outcome of the fasting insulin $(\mathrm{mUI} / \mathrm{ml}) \times$ glucose $(\mathrm{mmol} / \mathrm{l}) / 22.5$ was 3.0 . This result indicated no insulin resistance. These findings excluded the diagnosis of insulin resistance, T2D, Cushing's syndrome and hyperandrogenism.

$\mathrm{X}$-ray examination (done at 14 years old) revealed no abnormalities (Fig. 3).

As genetic mutations have been recognized in several cases of syndromic AN, a mutational analysis was performed in the proband and parents. Written informed consent was signed by the proband and her parents.

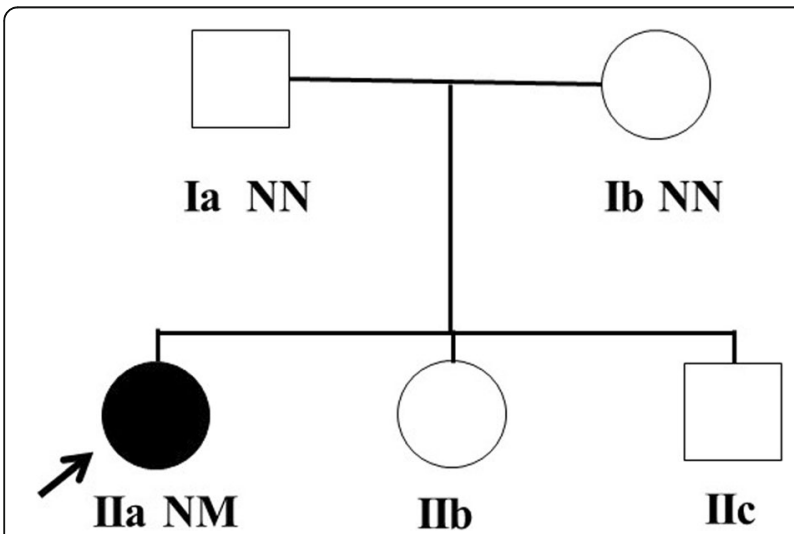

Fig. 1 Pedigree of family. Squares represent male family members, while circles represent female family members. Black symbol represents individual with acanthosis nigricans, blank symbols represent normal individuals. Arrow indicates proband in the family (lla). Variant carrier status present as N: Normal allele and M: Mutation. The sequence data displayed heterozygous mutation in FGFR3 (c.1949A > C, p.Lys650Thr) in the proband (Ila)

\section{Genetic analysis}

Peripheral blood samples $(4 \mathrm{ml})$ of the proband and her parents were collected. Genomic DNA was extracted from the blood using a QIAamp DNA Mini Kit (Qiagen China Co., Ltd., Shanghai, China) according to the manufacturer's recommendations. We first performed whole exome sequencing for the proband. Next, based on the test results of whole exome sequencing, the presence of the mutation in the proband and her parents was confirmed with direct Sanger sequencing of the affected exon.

\section{Whole exome sequencing}

All coding exons were enriched using the xGen Exome Research Panel v1.0 (Integrated DNA Technology, Inc). Captured DNA libraries were sequenced on Illumina Hiseq X Ten according to the manufacturer's instructions for paired-end $150 \mathrm{bp}$ reads. Variants were considered as pathogenic mutations if they exhibited the following components: i) rare or absent in the above genome databases; ii) variation expected to have a drastic effect on the protein (nonsense mutation, frame shift mutation, mutation at a splice site, or missense mutation is highly conserved among species); and iii) variation predicted to be damaging.

\section{Sanger sequencing to validate}

Sanger sequencing of the affected exon in FGFR3 was performed on DNA samples from the proband and her parents. According to the DNA sequence of the FGFR3 gene, primers of exon 14 of FGFR3 were designed using Primer Premier 5 software. The functional effects of protein variants were predicted by PolyPhen2 (http://genetics.bwh.harvard.edu/pph2/), SIFT (http://sift.jcvi.org) and Mutation Taster (http://www.mutationtaster.org).

Through data mining, combined with genetic characteristics and clinical manifestations, we identified a heterozygous c.1949A > C, p.Lys650Thr mutation in FGFR3 of the proband, which is considered to be a pathogenic mutation. As the proband's parents did not carry the mutation, the mutation identified in the proband was a de novo mutation. Sanger sequencing confirmation is shown in Fig. 4. The mutation caused change in the protein from $\mathrm{K}$ to $\mathrm{T}$ at p. Lys650, which is located in exon 14 of FGFR3. The pathogenicity of the mutation on bone and skin has been previously reported [1-6] and was confirmed using 3 different software programmes (The expected score scales of the mutation from each software programme are shown in Additional file 1: Table S1): SIFT (0), PolyPhen-2 (1) and Mutation taster (disease-causing).

\section{Discussion and conclusions}

In this study, we have identified the first Chinese general AN case caused by the mutation in FGFR3 

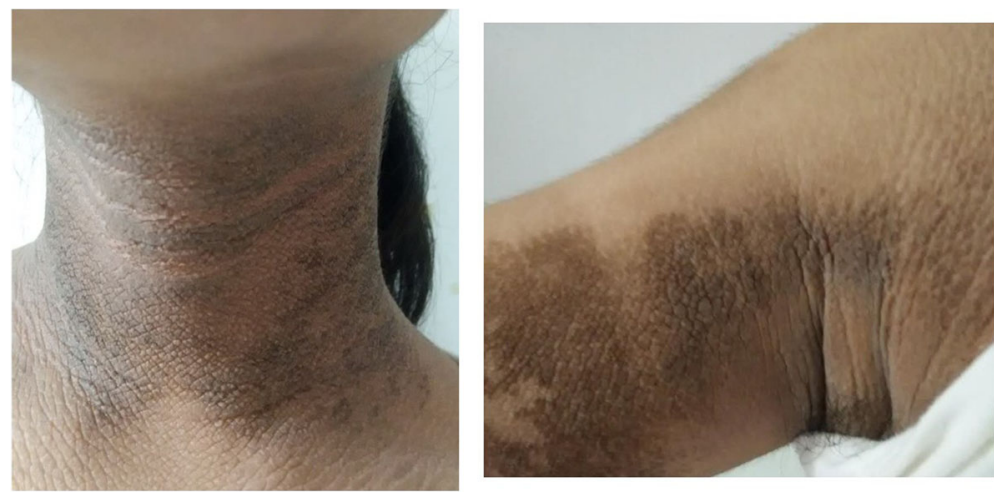

Fig. 2 Clinical images of the neck, and axillary fossa region of the case

Table 1 Laboratory investigation

\begin{tabular}{|c|c|c|}
\hline Laboratory (serum) & Value & Normal range \\
\hline Alanine transaminase $(\mathrm{U} / \mathrm{L})$ & 8 & $7-40$ \\
\hline Aspartate aminotransferase (U/L) & 16 & $13-35$ \\
\hline Total bilirubin (umol/L) & 9.1 & $5.1-22.2$ \\
\hline Direct bilirubin (umol/L) & 3.5 & $0-6.8$ \\
\hline Albumin (g/L) & 48 & $35-52$ \\
\hline Creatinine (umol/L) & 57 & $18-69$ \\
\hline Uric acid (umol/L) & 337 & $150-357$ \\
\hline Total cholesterol (mmol/L) & 4.03 & $2.85-5.7$ \\
\hline Triglyceride (mmol/L) & 0.36 & $0.45-1.70$ \\
\hline $\mathrm{HDL}-\mathrm{C}(\mathrm{mmol} / \mathrm{L})$ & 1.38 & $0.93-1.81$ \\
\hline LDL-C (mmol/L) & 2.24 & $<3.37$ \\
\hline Fating glucose (mmol/L) & 4.9 & $3.9-6.1$ \\
\hline Fasting insulin (ulU/ml) & 13.78 & $5.2-17.2$ \\
\hline HOMA-IR & 3.0 & $<3.0$ \\
\hline Glycosylatedhemoglobin (\%) & 5.1 & $4.5-6.3$ \\
\hline Homocysteine (umol/L) & 10.3 & $<15$ \\
\hline $\mathrm{hsCRP}(\mathrm{mg} / \mathrm{L})$ & 0.12 & $0-3.0$ \\
\hline Erythrocyte sedimentation rate $(\mathrm{mm} / \mathrm{h})$ & 5 & $0-20$ \\
\hline Cortisol (ug/dl) & 22.06 & $4-22.3$ \\
\hline Testosterone (ng/dl) & 17.3 & $25.6-42.6$ \\
\hline DHES (ug/dl) & 161.3 & $17-343$ \\
\hline $17 \mathrm{aOHP}(\mathrm{ng} / \mathrm{ml})$ & 1.04 & $0.27-2.9$ \\
\hline FT3 (pg/ml) & 3.27 & $1.8-4.1$ \\
\hline FT4 (ng/dl) & 1.231 & $0.81-1.89$ \\
\hline TSH (ulU/ml) & 3.242 & $0.38-4.34$ \\
\hline A-Tg (IU/ml) & $<10$ & $<115$ \\
\hline A-TPO (IU/ml) & $<5$ & $<34$ \\
\hline
\end{tabular}

Abbreviation: $H D L-C$ high-density lipoprotein cholesterol, $L D L-C$ low-density lipoprotein cholesterol, HOMA-IR homeostasis model assessment of insulin resistance, $h S C R P$ high sensitivity $C$ reactive protein, DHES dehydroepiandrosterone sulfate
(c.1949A > C, p.Lys650Thr). Additionally, we reviewed the previously reported cases due to this mutation.

AN is characterized by dark-brownish hyperpigmentation, velvety with thickening of the skin, and the skin lesions are usually founded in skin folds such as the neck, armpits, forehead, and groin. The exact incidence of AN is still unknown. AN's prevalence in whites is less than $1 \%$, whereas the prevalence is higher in dark-skinned people, approximately $13.3 \%$ [7]. AN in childhood is not rare and the benign hereditary form and the type associated with insulin resistance are commonly seen [8]. However, in the present case, the patient did not have insulin resistance or a family history of AN. Additionally, AN is correlated with numerous genetic syndrome, which can be divided into insulin resistance syndromes and fibroblast growth factor (FGF) defects [9]. The pathogenic variant (p.Lys650Thr) in FGFR3 has been recognized to be correlated with AN [1-6]. The present case is the first-reported Chinese patient having AN with this specific mutation.

FGFR3 is located at $4 \mathrm{p} 16.3$, and encodes the fibroblast growth factor receptor 3 [6]. The gene is highly conserved between members, and acts as a repressor of long bones growth [6]. The full-length FGFR3 protein consists of an extracellular region, a single hydrophobic membrane-spanning segment and a cytoplasmic tyrosine kinase domain. The extracellular portion of the protein combined with fibroblast growth factors; set in motion a cascade of downstream signals, influencing mitogenesis and differentiation, and ultimately playing a role in the development and maintenance of bone. Mutations in this gene lead to craniosynostosis and multiple types of skeletal dysplasia (https://www.ncbi.nlm.nih.gov/protein/4503711). Located in the tyrosine kinase domain II of FGFR3, P. Lys650 is an important residue for the biological function of FGFR3 [2]. Germline K650 T mutation can results in constitutive activation of FGFR3 signals through the activation of STAT1 and MEK/MAPK pathways, both of which are described relevant to the 

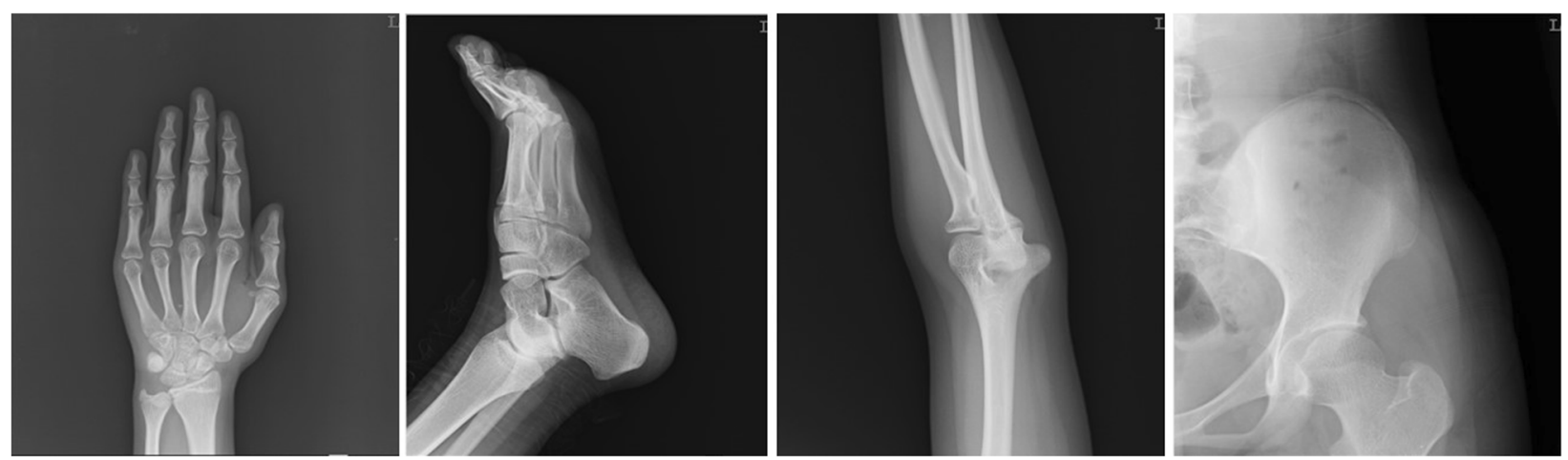

Fig. $3 X$ ray of the case (done at 14 years old, left side)

phenotypic consequences of skeletal dysplasia [10]. However, the exact mechanisms of the association between $\mathrm{AN}$ and the K650 T mutation have not been thoroughly elucidated to date. Given that $\mathrm{HCH}$ accompanying AN happens in subjects with p.Lys650Thr mutation, it has been speculated that the activation of the MAPK pathway (affecting proliferation of keratinocytes) and PI3-K/Akt pathway (affecting the expansion of the epidermal compartment) may play a role in the development of AN [2].

We reviewed the previously reported cases caused by a mutation in FGFR3. The specific mutation in FGFR3 was first reported in a family that included 4 patients diagnosed with AN without apparent skeletal deformity [6]. Currently, 7 pedigrees containing 27 cases (including the current case) have been described (Table 2) [1-6]. The reviewed cases yield no gender differences, and the male to female ratio was 13:14. The height of all of the patients tends to be low, which is consistent with the case presented in our study. The majority of the individuals showed excessive skin pigmentation without obesity or diabetes mellitus (with the exception of patient 5 who was diagnosed with adult onset diabetes mellitus) during infancy. Moreover, 19 of the 27 cases were diagnosed as $\mathrm{HCH}$ plus $\mathrm{AN}$, while several cases were described

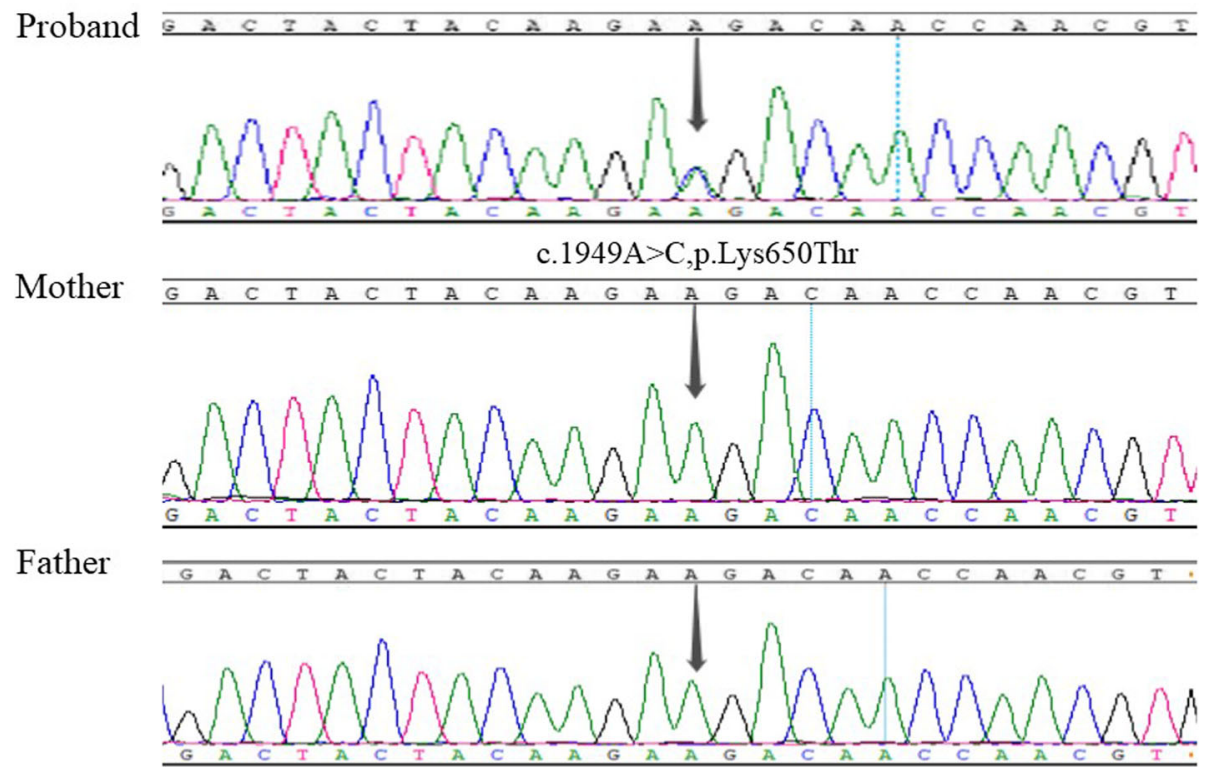

Fig. 4 Sequencing of exon 14 of FGFR3 (NM_000142). A heterozygous C.1949A > C (p.Lys650Thr) at codon 650 of FGFR3gene was revealed in the proband, while not in her unaffected parents 
Table 2 Reports on AN with or without HCH due to p. Lys650Thr of FGFR3

\begin{tabular}{|c|c|c|c|c|c|c|c|c|c|c|c|c|}
\hline Patients & Onset age & Age & Gender & AN & $\mathrm{HCH}$ & Birth weight (g) & Height (cm) & $\mathrm{BMl}$ & FBG(74-105 mg/dL) & FINS $(1.5-18.5 \mu \mathrm{U} / \mathrm{ml})$ & HOMA-IR & $\mathrm{HbA} 1 \mathrm{c}$ \\
\hline \multicolumn{13}{|c|}{ Family 3[6] } \\
\hline $1^{a}$ & infancy & 4 & $\mathrm{~F}$ & + & - & / & 105.5 (5.5y,8th) & 15.5 & normal & / & / & normal \\
\hline 2 & / & 25 & M & + & - & / & 156 & 19.7 & / & / & / & / \\
\hline 3 & / & 29 & $\mathrm{~F}$ & + & - & / & 145 (<5th) & 30.5 & / & / & / & / \\
\hline 4 & / & 11 & $\mathrm{~F}$ & + & - & / & 139 (26th) & 21.2 & / & / & / & / \\
\hline \multicolumn{13}{|c|}{ Family 1[2] } \\
\hline 5 & infancy & 51 & M & + & + & / & 144.3 & 26.4 & 190 & 5.3 & 2.4 & 7.9 \\
\hline 6 & infancy & 49 & $\mathrm{~F}$ & + & + & / & 141.2 & 28.1 & 81 & 3.6 & 0.7 & 4.4 \\
\hline 7 & infancy & 46 & $\mathrm{~F}$ & + & + & / & 145.5 & 26.9 & 92 & 4.4 & 0.9 & 4.9 \\
\hline 8 & infancy & 40 & $\mathrm{~F}$ & + & + & / & 149.4 & 28.3 & 87 & 5.1 & 1.1 & 4.8 \\
\hline 9 & infancy & 35 & $\mathrm{~F}$ & + & + & / & 153 & 25.8 & 85 & 3.9 & 0.8 & 4.3 \\
\hline $10^{\mathrm{a}}$ & infancy & 16 & M & + & + & 3515 & / & 24.2 & 97 & 7.0 & 1.6 & 4.6 \\
\hline 11 & infancy & 18 & $\mathrm{~F}$ & + & + & / & 147.9 & 26.5 & 73 & 2.8 & 0.5 & 4.2 \\
\hline 12 & infancy & 8 & M & + & + & / & / & 20.6 & 88 & 3.8 & 0.8 & 4.5 \\
\hline 13 & infancy & 13 & M & + & + & / & / & 20.2 & 90 & 6.9 & 1.5 & 4.6 \\
\hline 14 & infancy & 7 & M & + & + & / & / & 23 & 92 & 6.1 & 1.3 & 4.7 \\
\hline \multicolumn{13}{|c|}{ Family 5[1] } \\
\hline $15^{\mathrm{a}}$ & 3 & 15 & $\mathrm{~F}$ & + & - & / & / & / & / & / & / & / \\
\hline 16 & / & / & $\mathrm{F}$ & + & - & / & / & / & / & / & / & / \\
\hline 17 & / & / & $\mathrm{F}$ & + & - & / & / & / & / & / & / & / \\
\hline \multicolumn{13}{|c|}{ Family 2[3] } \\
\hline $18^{\mathrm{a}}$ & 12 & 14 & M & + & + & / & 143 & 21.5 & I & / & / & / \\
\hline 19 & / & / & M & + & + & / & / & / & I & / & / & / \\
\hline 20 & / & / & M & + & + & / & / & / & / & / & / & / \\
\hline 21 & / & / & M & + & + & / & / & / & / & / & / & / \\
\hline \multicolumn{13}{|c|}{ Family 4[4] } \\
\hline $22^{a}$ & 1 & 3 & M & + & + & 2883 & 91.7 (3-10th) & 19.4 & 76 & 8.8 & 1.6 & \\
\hline \multicolumn{13}{|c|}{ Family 6[5] } \\
\hline $23^{\mathrm{a}}$ & 2 & 10 & M & + & + & / & 128 & 23.1 & / & 3.1 & / & / \\
\hline 24 & / & 12 & $\mathrm{~F}$ & + & + & / & 135 & 23.5 & / & / & / & / \\
\hline 25 & / & 47 & M & + & + & / & 155 & 25 & / & / & / & / \\
\hline 26 & / & 74 & $\mathrm{~F}$ & + & + & / & 140 & 23 & / & / & / & / \\
\hline \multicolumn{13}{|c|}{ Current case } \\
\hline $27^{a}$ & 4 & 17 & $\mathrm{~F}$ & + & - & 4000 & 146 & 24.4 & 88.2 & 13.78 & 3.0 & 5.1 \\
\hline
\end{tabular}

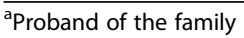

AN acanthosis nigricans, $H C H$ hypochondroplasia, BMI body mass index, FBG fasting glucose, FINS fasting insulin, HOMA-IR homeostasis model assessment index of insulin resistance, $\mathrm{HbA1C}$ glycosylated hemoglobin

as "pure" familial AN $(n=8)$. Additionally, apart from the familial AN, few cases harbor a de novo mutation [4]. In our study, neither of the parents of the patient carried the FGFR3 mutation, indicating a de novo mutation in the proband.

The treatment of AN should focus on correction of the underlying pathological state. Currently, there is no good treatment for AN caused by a mutation in FGFR3. It may be necessary for the present patient to monitor the levels of fasting blood glucose and androgen regularly.

In summary, we report the first Chinese case of AN with p.Lys650Thr mutation in FGFR3, demonstrating a widespread skin pigmentation, and short stature. FGFR3 sequencing is a feasible approach to identify the aetiology of AN, and the effects of FGFR3 on bone and skin should be further analysed. 


\section{Additional file}

Additional file 1: Table S1. The expected score scale of the mutation from each software. (DOCX $13 \mathrm{~kb}$ )

\section{Abbreviations}

AN: Acanthosis nigricans; FGFR3: Fibroblast growth factor receptor 3; $\mathrm{HCH}$ : Hypochondroplasia

\section{Acknowledgements}

The authors would like to thank the participants in this study.

\section{Funding}

This work was supported by the grants from National Natural Science Foundation of China (No. 81170736, 81570715, 81870579). These funding bodies had role in the design of the study and collection, analysis, and interpretation of data and in writing the manuscript.

\section{Availability of data and materials}

All data generated or analyzed during this study are included in the published article.

\section{Authors' contributions}

JF analyzed the clinical and experimental data and wrote the manuscript; $Y Z$ conducted the gene analysis and was a major contributor to the writing of the manuscript. TW and QZ contributed with interpretation of data and critical reviews of the article. XX was contributed to acquisition and interpretation of the data, and revised the manuscript. All authors read and approved the final manuscript.

\section{Ethics approval and consent to participate}

The study was approved by the ethics committee of Peking Union Medical College Hospital, China. The reference number is B137 and date of approval is 2015-6-29. Written informed consent was obtained from the patient and her parents.

\section{Consent for publication}

The patient and her parents have signed a written informed consent to use all the familial members' personal, medical and clinical details for research and to publish related scientific findings.

\section{Competing interests}

The authors declare that they have no competing interests.

\section{Publisher's Note}

Springer Nature remains neutral with regard to jurisdictional claims in published maps and institutional affiliations.

\section{Author details}

${ }^{1}$ Department of Endocrinology, Chinese Academy of Medical Sciences and Peking Union Medical College, Peking Union Medical College Hospital, Beijing, China. ${ }^{2}$ Department of Center of PET-CT, Chinese Academy of Medical Sciences Cancer Institute and Hospital, Beijing 100021, China.

Received: 27 September 2018 Accepted: 7 January 2019

Published online: 11 January 2019

\section{References}

1. Ichiyama S, Funasaka Y, Otsuka Y, Takayama R, Kawana S, Saeki H, Kubo A. Effective treatment by glycolic acid peeling for cutaneous manifestation of familial generalized acanthosis nigricans caused by FGFR3 mutation. J Eur Acad Dermatol Venereol. 2016;30(3):442-5.

2. Castro-Feijoo L, Loidi L, Vidal A, Parajes S, Roson E, Alvarez A, Cabanas P, Barreiro J, Alonso A, Dominguez F, et al. Hypochondroplasia and Acanthosis nigricans: a new syndrome due to the p.Lys650Thr mutation in the fibroblast growth factor receptor 3 gene? Eur J Endocrinol. 2008;159(3):243-9.

3. Cossiez Cacard MA, Coulombe J, Bernard P, Kaci N, Bressieux JM, Souchon PF, Motte J, Legeai-Mallet L, Hadj-Rabia S, Eschard C. Familial hypochondroplasia and acanthosis nigricans with FGFR3 K650T mutation. J Eur Acad Dermatol Venereol. 2016:30(5):897-8.

4. Tokura $Y$, Hirai H, Hamada J, Hasegawa K, Ishii E. Acanthosis nigricans in a Japanese boy with hypochondroplasia due to a K650T mutation in FGFR3. Dermatol. 2017;26(4):223-8.

5. Yasuda M, Morimoto N, Shimizu A: Familial acanthosis nigricans with the FGFR3 mutation: differences of pigmentation between male and female patients. 2018.

6. Berk DR, Spector EB, Bayliss SJ. Familial acanthosis nigricans due to K650T FGFR3 mutation. Arch Dermatol. 2007;143(9):1153-6

7. Kutlubay Z, Engin B, Bairamov O, Tuzun Y. Acanthosis nigricans: a fold (intertriginous) dermatosis. Clin Dermatol. 2015;33(4):466-70.

8. Inamadar AC, Palit A. Generalized acanthosis nigricans in childhood. Pediatr Dermatol. 2004;21(3):277-9.

9. Torley D, Bellus GA, Munro CS. Genes, growth factors and acanthosis nigricans. Br J Dermatol. 2002;147(6):1096-101.

10. Ornitz DM. FGF signaling in the developing endochondral skeleton. Cytokine Growth Factor Rev. 2005;16(2):205-13.
Ready to submit your research? Choose BMC and benefit from:

- fast, convenient online submission

- thorough peer review by experienced researchers in your field

- rapid publication on acceptance

- support for research data, including large and complex data types

- gold Open Access which fosters wider collaboration and increased citations

- maximum visibility for your research: over $100 \mathrm{M}$ website views per year

At $\mathrm{BMC}$, research is always in progress.

Learn more biomedcentral.com/submission 